\title{
OPTICAL MULTISTABILITY FROM FOUR-WAVE MIXING IN A RESONANT MEDIUM
}

\author{
V.V. KABANOV, A.S. RUBANOV \\ Institute of Physics, BSSR Academy of Sciences, 220602 Minsk, USSR
}

\author{
A.L. TOLSTIK and A.V. CHALEY \\ Physics Department, Byelorussian State University, 220080 Minsk, USSR
}

Received 18 July 1988; revised manuscript received 15 December 1988

\begin{abstract}
A theoretical study is made of the conditions for bifurcation and optical multistability at degenerate FWM in a resonant medium taking into account nonlinear absorption and energy pumping between all interacting waves.
\end{abstract}

\section{Introduction}

In recent years much consideration is being given to resonatorless optical bistability (OB). It was predicted for structures with distributed feedback [1] and at four-wave mixing (FWM) in a cubic nonlinearity medium [2] (see also refs. [3,4]). Works $[5,6]$ are devoted to theoretical and experimental studies of $O B$ and hysteresis at six-wave mixing in a CdS crystal on fifth-order nonlinearity. OB at FWM was considered in photorefractive crystals [7] as well as in a two-level resonant medium as a purely phase modulation approximation [8]. In refs. [9,10], FWM was analyzed for reference and signal fields polarized both orthogonally and in parallel and the possibility of obtaining multistability was demonstrated.

The present paper deals with a theoretical study of the conditions for bifurcations and realization of optical multistability (OM) at degenerate FWM in a resonant medium taking into account the nonlinear absorption and energy pumping between all the interacting waves.

\section{The theoretical model}

Considering the two-level model of a resonant medium, the light-induced change in the refractive in- dex $\Delta \hat{n}(\omega, \mathscr{E})=\hat{n}(\omega, \mathscr{E})-\hat{n}_{0}(\omega)$ can be expressed as [11]

$\Delta \hat{n}(\omega, \mathscr{E})=-\hat{\alpha} \kappa_{0} I /(1+\alpha I)$,

where $\hat{n}_{0}(\omega)=n_{0}(\omega)+i \kappa_{0}(\omega)$ is the complex refractive index of the unexcited medium, $I=c n_{0}|\mathscr{E}|^{2} /$ $4 \pi$ is the field intensity, $\mathscr{E}=\sum_{j} \mathscr{E}_{j}$ of the interacting waves (the pump $\mathscr{E}_{ \pm}=\frac{1}{2} E_{ \pm} \exp \left[\mathrm{i}\left(\boldsymbol{k}_{ \pm} \boldsymbol{r}_{1}-\omega t\right)\right]+$ c.c., and the signal and conjugate waves $\mathscr{E}_{\mathrm{s}, \mathrm{c}}=$ $\frac{1}{2} E_{\mathrm{s}, \mathrm{c}} \exp \left[\mathrm{i}\left(\boldsymbol{k}_{\mathrm{s}, \mathrm{c}} \boldsymbol{r}_{2}-\omega t\right)\right]+$ c.c. $)$. The complex parameter of nonlinearity is determined by this expression

$\hat{\alpha}=a+\mathrm{i} \alpha=\left(\hat{\theta}_{12}+\hat{\theta}_{21}\right) / v \rho_{21}$,

where $\hat{\theta}_{i j}(\omega)=\theta_{i j}(\omega)+\mathrm{i} B_{i j}(\omega), \theta_{i j}(\omega)$ are related to the Einstein coefficient $B_{i j}(\omega)$ through the dispersion ratios, $\rho_{21}$ is the probability of spontaneous and nonradiative transition, $v$ is the velocity of light in the medium. A spectroscopic model like this can be used to describe both atomic media and these with a complicated form of spectra that exhibit a Stokes shift of absorption and emission bands such as colored crystals or dye solutions [12].

The nonlinear polarization can be written as

$$
\mathscr{P}_{\mathrm{NL}}=\chi_{\mathrm{NL}}=-\frac{\kappa_{0} \hat{n}_{0} \hat{\alpha} I}{2 \pi(1+\alpha I)} \mathscr{E} \text {. }
$$

At coplanar FWM the vectors of all the dynamic gratings (DG) induced in the medium can be ex- 
pressed as a linear combination of only two DG with linearly independent vectors, for example, $\mathscr{K}_{x}=$ $\boldsymbol{k}_{+}+\boldsymbol{k}_{\mathrm{s}}$ and $\mathscr{X}_{y}=\boldsymbol{k}_{+}-\boldsymbol{k}_{\mathrm{s}}$ as shown in fig. 1. To describe the process of FWM taking into account the nonlinear absorption and effective energy pumping between the interacting waves, we have used expansion of the nonlinear susceptibility $\chi_{\mathrm{NL}}$ in series of the harmonics of these two DG, which, under the conditions of Bregg's reflection, has permitted writing the following system of reduced wave equations [9]:

$$
\begin{gathered}
\left.\partial E_{ \pm} / \partial r_{1}= \pm(\mathrm{i} 2 \pi \omega) / c n_{0}\right)\left(\chi_{0.0} E_{ \pm}\right. \\
\left.+\chi_{ \pm 1 . \pm 1} E_{\mp}+\chi_{0 . \pm 1} E_{\mathrm{s} . \mathrm{c}}+\chi_{ \pm 1.0} E_{\mathrm{c}, \mathrm{s}}\right) \\
\partial E_{\mathrm{s} . \mathrm{s}} / \partial r_{2}= \pm\left(\mathrm{i} 2 \pi \omega / c n_{0}\right)\left(\chi_{0, \mp 1} E_{ \pm}\right. \\
\left.+\chi_{ \pm 1.0} E_{\mp}+\chi_{0,0} E_{\mathrm{s}, \mathrm{c}}+\chi_{ \pm 1 . \mp 1} E_{\mathrm{c} . \mathrm{s}}\right) .
\end{gathered}
$$

Here

$$
\begin{aligned}
& \chi_{m, n}=\left(4 \pi^{2}\right)^{-1} \int_{-\pi}^{\pi} \int_{-\pi}^{\pi} \chi_{\mathrm{NL}}\left(\xi_{x}, \xi_{y}\right) \\
& \times \exp \left[\mathrm{i}\left(m \xi_{x}+n \xi_{y}\right)\right] \mathrm{d} \xi_{x} \mathrm{~d} \xi_{y}, \\
& \xi_{x}=\left|\mathscr{K}_{x}\right| x, \quad \xi_{y}=\left|\mathscr{K}_{y}\right| y .
\end{aligned}
$$

The Fourier-expansion components $\chi_{ \pm 1, \pm 1}, \chi_{ \pm 1, \mp 1}$ are responsible, respectively, for the self-diffraction of the reference, the signal and the conjugate field. The components $\chi_{0, \pm 1}, \chi_{ \pm 1.0}$ take into account the processes of both self-diffraction and parametric diffraction, and $\chi_{0,0}$ describes the amplitude absorption of interacting waves.

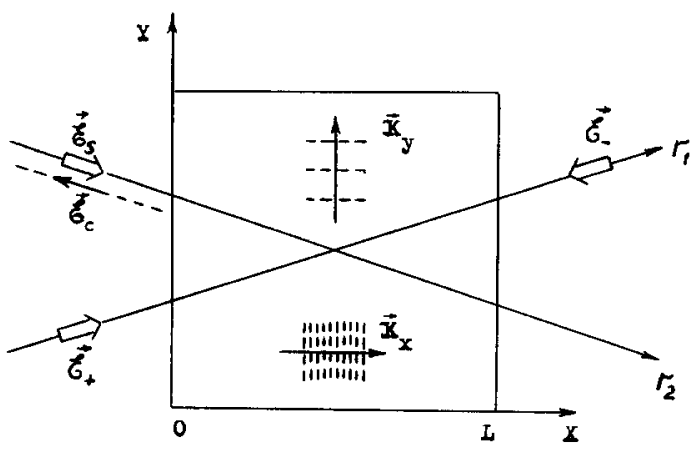

Fig. 1. Schematic diagram of coplanar FWM.

\section{Bifurcation and optical multistability}

For practically collinear FWM of the fields polarized in one plane the system of equations (4), (5) can be represented as

$$
\begin{aligned}
& \frac{\partial E_{+, \mathrm{c}}}{\partial x}=\mp \frac{\mathrm{i} \omega \kappa_{0}}{c}\left[\frac{a}{\alpha} E_{+, c}\right. \\
&\left.-\frac{\hat{\alpha}}{\pi \alpha} \int_{-\pi}^{\pi} \frac{E_{+, \mathrm{c}}+E_{\mathrm{c},+} \exp \left(\mp \mathrm{i} \xi_{x}\right)}{A_{0}\left(1+A_{0}-J_{+}+J_{-}\right)} \mathrm{d} \xi_{x}\right], \\
& \frac{\partial E_{-, \mathrm{s}}}{\partial x}= \pm \frac{\mathrm{i} \omega \kappa_{0}}{c}\left[\frac{a}{\alpha} E_{-, \mathrm{s}}\right. \\
&\left.-\frac{\hat{\alpha}}{\pi \alpha} \int_{-\pi}^{\pi} \frac{E_{-, s}+E_{\mathrm{s},-} \exp \left( \pm \mathrm{i} \xi_{x}\right)}{A_{0}\left(1+A_{0}+J_{+}-J_{-}\right)} \mathrm{d} \xi_{x}\right],
\end{aligned}
$$

where

$$
\begin{aligned}
& A_{0}=\left[1+2\left(J_{+}+J_{-}\right)+\left(J_{+}-J_{-}\right)^{2}\right]^{1 / 2}, \\
& J_{ \pm}=\underline{\alpha}\left|E_{ \pm}+E_{\mathrm{c}, \mathrm{s}} \exp \left(\mp \mathrm{i} \xi_{x}\right)\right|^{2}, \\
& \underline{\alpha}=c n_{0} \hat{\alpha} / 8 \pi .
\end{aligned}
$$

In the case of orthogonally polarized reference $E_{ \pm}$ and signal $E_{\mathrm{s}, \mathrm{c}}$ fields (FWM in the absence of smallscale gratings) the system of equations is simplified

$$
\begin{aligned}
& \frac{\partial E_{+, \mathrm{c}}}{\partial x}= \pm \frac{\mathrm{i} \omega \kappa_{0}}{c}\left[\frac{\hat{\alpha}-a A_{1}}{\alpha} E_{+, \mathrm{c}}\right. \\
& \left.-\frac{2 \underline{\hat{\alpha}\left(E_{+, \mathrm{c}}\left|E_{\mathrm{s},-}\right|^{2}+E_{\mathrm{s},-} E_{\mathrm{c},+} E_{-, \mathrm{s}}^{*}\right)}}{1+A_{1}+\alpha I_{\Sigma}}\right], \\
& \frac{\partial E_{-, \mathrm{s}}}{\partial x}=\mp \frac{\mathrm{i} \omega \kappa_{0}}{c}\left[\frac{\hat{\alpha}-a A_{1}}{\alpha} E_{-, \mathrm{s}}\right. \\
& \left.-\frac{2 \hat{\alpha}\left(E_{-. \mathrm{s}}\left|E_{\mathrm{c},+}\right|^{2}+E_{\mathrm{s},-} E_{\mathrm{c},+} E_{+, \mathrm{c}}^{*}\right)}{1+A_{1}+\alpha I_{\Sigma}}\right],
\end{aligned}
$$

where

$$
\begin{aligned}
& I_{\Sigma}=\sum_{j} I_{j}, \\
& \quad A_{1}=\left[\left(1+\alpha I_{\Sigma}\right)^{2}-4 \underline{\alpha}^{2}\left|E_{+} E_{\mathrm{s}}^{*}+E_{-}^{*} E_{\mathrm{c}}\right|^{2}\right]^{1 / 2} .
\end{aligned}
$$

A similar system of equations for the two-level model of a medium with coinciding absorption and emission lines which takes into account only small-scale gratings was considered in ref. [13]. But multistability was not studied in that paper.

Numerical analysis of the systems of equations (6), 
(7) and (8), (9) has made it possible to study the FWM process taking into account the changes of all interacting waves in the nonlinear medium volume and to determine the conditions imposed on the light fields and parameters of the nonlinear layer under which resonatorless OM manifests itself. The multistability regime is due to the predominating contribution of the phase dynamic gratings and it can be realized by detuning the frequency of interacting waves in both directions from the absorption band centre at coinciding absorption and emission lines and towards the short-wave region at Stokes shift of the bands [14]. For media with the Lorentz shape of the line detuning by at least $\sim 3.5$ halfwidth is required whereas for gaussian-profile approximated spectra detuning by $\sim 1.4$ halfwidth is sufficient. At the same time the optical density of the medium must be higher than the critical value determined as a linear approximation by signal fields [11].

The main features of OM at FWM illustrated in figs. 2, 3, 4 for orthogonally polarized fields $E_{+.-}$, $E_{\text {s.c }}$ hold for interacting waves polarized in one plane (fig. 5).

The picture of FWM as a nonlinear process can be traced in fig. 2 which shows the reflection coefficient

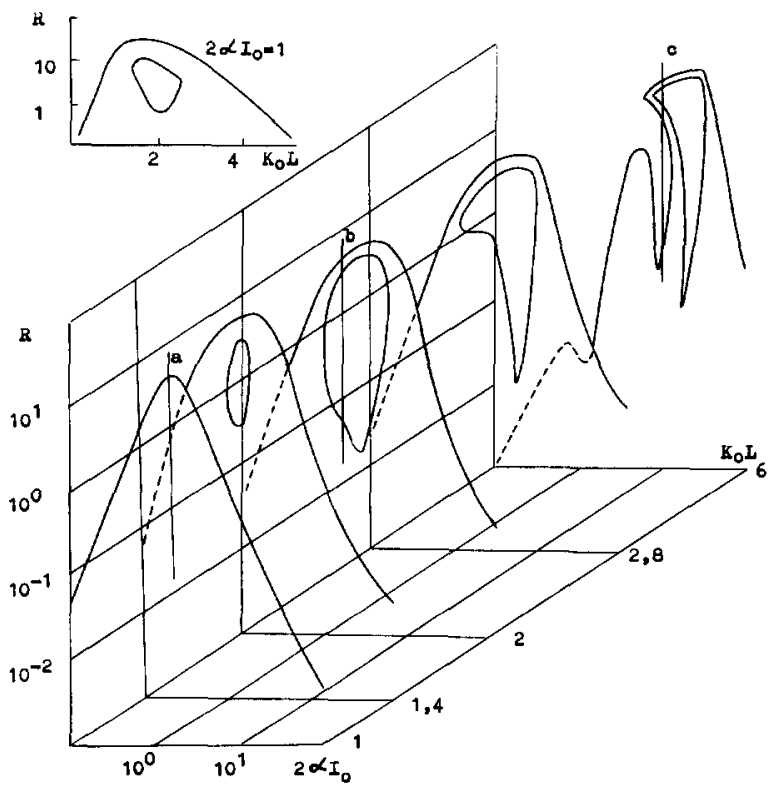

Fig. 2. The reflection coefficient $R$ versus the integrated intensity of reference waves $2 \alpha I_{0}$ and optical density of the nonlinear me$\operatorname{dium} K_{0} L$.

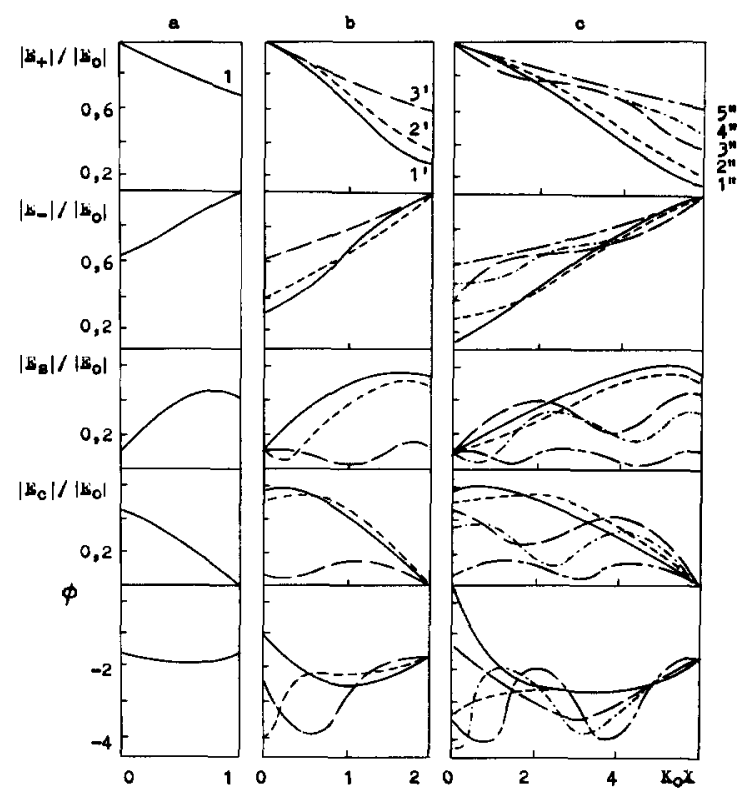

Fig. 3. The distribution of the amplitudes and phase mismatch of interacting waves in the nonlinear medium volume.

of the phase-conjugate wave $R=\left|E_{\mathrm{c}}(0) / E_{\mathrm{s}}(0)\right|^{2}$, $\left(E_{\mathrm{c}}(L)=0\right)$ versus the integrated intensity of the reference waves $2 \alpha I_{0}, \quad\left(I_{+}(0)=I_{-}(L)=I_{0}\right.$ $\left.=c n_{0}\left|E_{0}\right|^{2} / 8 \pi\right)$ incident on the nonlinear cell and the optical density of the medium $K_{0} L,\left(K_{0}=2 \omega \kappa_{0} /\right.$ $c)$. It has been calculated for radiation frequency detuning by $\eta=\left(\omega-\omega_{12}\right) / \Delta=2$ from the centre $\omega_{12}$ of the gaussian line of the absorption band with halfwidth $\Delta$, for a Stokes shift $\delta=\left(\omega_{21}-\omega_{12}\right) / \Delta=-1.6$ ( $\omega_{21}$ is the centre of the line of the emission band) and a signal intensity to pump intensity ratio at the input $I_{\mathrm{so}} / I_{0}=10^{-2}$. At small optical densities the system of equations (8), (9) or (6), (7) has a unique solution (indicated by a vertical line (a)). With increasing $K_{0} L$ the FWM process can pass through the bifurcation point lying in the region of the resonant medium saturation intensity $I_{\mathrm{sat}}=\alpha^{-1}$. Then the system will have three solutions (see, for example, $b$ ). The described behaviour is illustrated in the upper part of fig. 2 which shows the section of a three-dimensional picture $R\left(I_{0}, K_{0} L\right)$ by the plane $2 \alpha I_{0}=1$. Further increase in the optical density gradually transforms the bistable region and leads to the appearance of the following bifurcation. In this case the number of solutions to the system increases to five (c). 
Individual stable states realized in the multistable regime of FWM differ as to the spatial distribution of the amplitudes and phases of the interacting waves (curves 1, 1', 1", 3', 3", 5" of fig. 3). At the same time one of the pair of solutions similar in spatial distribution is unstable (curves $2^{\prime}, 2^{\prime \prime}, 4^{\prime \prime}$ ). The criterion for stability of obtained solutions was assumed to be their stability to variation of the boundary conditions of the problem. The distributions of the amplitudes and the phase mismatch of interacting waves $\phi=\varphi_{+}+\varphi_{-}-\varphi_{\mathrm{s}}-\varphi_{\mathrm{c}}$ in the nonlinear medium volume shown in fig. 3 correspond to the optical density $K_{0} L=1$ (a), 2 (b), 6 (c) and integrated intensity of pump waves $2 \alpha I_{0}=1.5(\mathrm{a}, \mathrm{b}), 7.5$ (c) indicated by vertical lines in fig. 2 . It is seen that with passing through the bifurcation point there remain solutions whose character of spatial distribution is analogous to the distribution at FWM in the absence of the bistable regime (cf. curves 1 and $1^{\prime}, 1^{\prime \prime}$ ). After the second bifurcation the similarity of spatial distribution for solutions 3' and 3" remains. At the same time there appear new solutions (higher-order modes) (curve $3^{\prime}$ after the first bifurcation and 5" after the second one). These solutions practically coincide with those obtained for a given pump as a linear approximation by signal fields [11]. Being in agreement with the least value of energetic efficiency of conjugate wave formation, they are characterized by a higher frequency of change of the signal and conjugate field amplitudes in the nonlinear medium volume which remain much smaller than the pump wave amplitudes.

The hysteresis behaviour for the signal and conjugate waves controlled by varying the input signal intensity manifest itself simultaneously with the hysteresis for the pump fields whose loop has a reverse behaviour (fig. 4). So, with increasing input signal intensity, when the signal and conjugate wave amplitudes transmitted through a nonlinear layer increase abruptly, the amplitudes of the pump waves decrease. In this case, the FWM process changes to the regime of effective energy pumping of reference waves to signal ones. This regime is reversed at signal intensities close to zero (fig. 4a). Under the conditions for the tristable regime of FWM, successive transitions of the system to two states with high efficiency are possible (fig. $4 b$ ).

An increase in the signal wave intensity at the in-
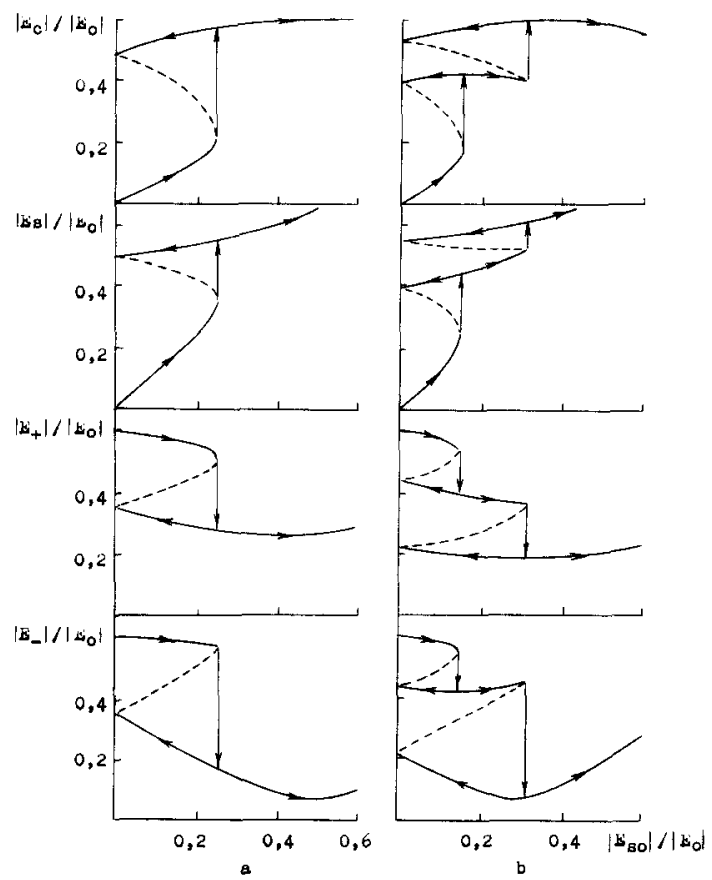

Fig. 4. The amplitudes of the conjugate $\left|E_{\mathrm{c}}\right|$, the signal $\left|E_{\mathrm{s}}\right|$ and the reference waves $\left|E_{ \pm}\right|$at the output from the nonlinear medium as a function of the signal amplitude at the input $\left|E_{50}\right|$ at optical density of the nonlinear layer $K_{0} L=2$ (a), 6 (b), $I_{\mathrm{s} 0}$ / $I_{0}=10^{-2}, \eta=2$.

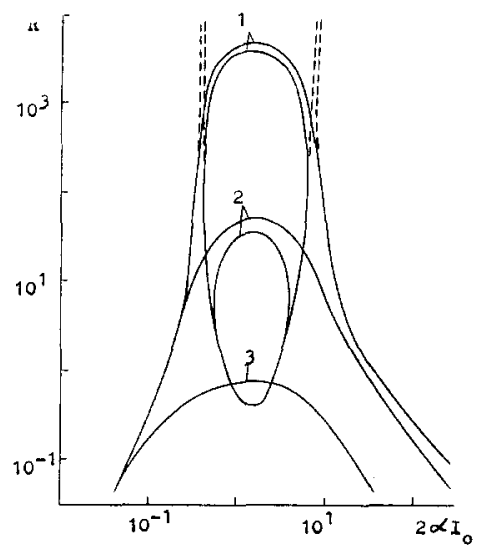

Fig. 5. The reflection coefficient $R$ as a function of the integrated intensity of reference waves $2 \alpha I_{0}$ for FWM of fields polarized in one plane at $\eta=2, K_{0} L=1, I_{50} / I_{0}=10^{-4}(1), 10^{-2}(2), 1$ (3).

put to the nonlinear cell leads to the disappearance of the multistable regime. This situation is illustrated in fig. 5 which shows the reflection coefficient versus the pump intensity at the input to the non- 
linear medium for various ratios $I_{\mathrm{s} 0} / I_{0}$. It is seen that the bistable state of the system occupies a limited region which is the larger, the smaller the ratio of the signal intensity to the reference wave intensity at the input to the nonlinear medium. With increasing $I_{\mathrm{s} 0} /$ $I_{0}$ this region reduces and at $I_{\mathrm{s} 0} / I_{0} \simeq 0.1$ it disappears. The broken line indicates the dependence $R\left(I_{0}\right)$ calculated as a linear approximation by signals with regard to the nonlinear absorption of all interacting waves.

\section{References}

[1] H.G. Winful, J.H. Marburger and E. Garmire, Appl. Phys. Lett. 35 (1979) 379.

[2] H.G. Winful and J.H. Marburger, Appl. Phys. Lett. 36 (1980) 613.

[3] R. Lytel, J. Opt. Soc. Am. 1B (1984) 91.
[4] K. Tajima and H. Hsu, Opt. Quantum Electron. 17 (1985) 149.

[5] A.A. Borshch, M.S. Brodin, V.I. Volkov and N.V. Kukhtarev, Sov. J. Quantum Electron. 8 (1981) 1304.

[6] A. Borshch, M. Brodin, V. Volkov, N. Kukhtarev and V. Starkov, J. Opt. Soc. Am. 1A (1984) 40.

[7] K.D. Shaw and M. Gronin-Golomb, Optics Comm. 65 (1988) 301.

[8] N. Kukhtarev, A. Borshch, M. Brodin, V. Volkov and T. Semenets, J. Physique 44 (1983) C2-5.

[9] V.V. Kabanov, A.S. Rubanov, A.L. Tolstik and A.V. Chaley, Vth All-Union Conf. on Holography, Abstracts, Riga (1985) 139.

[10] V.V. Kabanov, A.S. Rubanov, A.L. Tolstik and A.V. Chaley, preprint Institute of Physics, BSSR Academy of Sciences, N 411 (1986).

[11] V.V. Kabanov and A.S. Rubanov, Optics Comm. 44 (1983) 361.

[12] V.V. Kabanov and A.S. Rubanov, Acta Physica Hungarica 61 (1987) 99.

[13] W.P. Brown, J. Opt. Soc. Am. 73 (1983) 629.

[14] V.V. Kabanov, A.S. Rubanov, A.L. Tolstik and A.V. Chaley, Opt. Quantum Electron. 19 (1987) 351. 